\title{
LADDER DIAGRAMS AND WEAKLY SEPARATED SET-SYSTEMS
}

\section{I. DANILOV ${ }^{1}$, A. V. KARZANOV $2^{*}$ and G. A. KOSHEVOY ${ }^{1}$}

${ }^{1}$ Central Institute of Economics and Mathematics of the RAS

47, Nakhimovskii Prospect

117418 Moscow

Russia

e-mail: danilov@cemi.rssi.ru

koshevoy@cemi.rssi.ru

2Institute for System Analysis at Federal Research Center

Computer Science and Control of the RAS

9, Prospect 60 Let Oktyabrya

117312 Moscow

Russia

e-mail: sasha@cs.isa.ru

\begin{abstract}
Studying quasicommuting flag minors of a quantum matrix, Leclerc and Zelevinsky introduced the notion of weakly separated collections of subsets of the set $[n]=\{1, \ldots, n\}$. Answering their conjectures on such collections, there have been proved that some natural domains $\mathcal{D} \subseteq 2^{[n]}$, in particular, the Boolean cube $2^{[n]}$ and the discrete Grassmannian $\{X \subseteq[n]:|X|=m\}$ for
\end{abstract}

2010 Mathematics Subject Classification: 05C75, 05E99.

Keywords and phrases: quasicommuting quantum minors, weakly separated sets, strongly separated sets, lattice paths.

${ }^{*}$ Corresponding Author.

Received December 28, 2015

(C) 2015 Scientific Advances Publishers 
$m \in[n]$, possess the property of purity, which means that all inclusion-wise maximal weakly separated collections in $\mathcal{D}$ have the same size.

In this note, we prove the purity for a class of domains generalizing Boolean cubes and discrete Grassmannians. It is generated by so-called steep ladder diagrams.

\section{Introduction}

For a positive integer $n$, the set $\{1,2, \ldots, n\}$ with the usual order is denoted by $[n]$. For a subset $X \subseteq[n]$ formed by elements $x_{1}<x_{2}<\ldots<x_{k}$, we use notation $\left(x_{1}, \ldots, x_{k}\right)$ for $X, \min (X)$ for $x_{1}$, and $\max (X)$ for $x_{k}$, where $\min (X)=\max (X):=0$ if $X=\emptyset$.

There are several natural binary relations on the set $2^{[n]}$ of all subsets of $[n]$. Namely, for distinct $A, B \subseteq[n]$, we write:

(i) $A \prec B$ if $A=\left(a_{1}, \ldots, a_{k}\right), B=\left(b_{1}, \ldots, b_{m}\right), k \leq m$, and $a_{i} \leq b_{i}$ for $i=1, \ldots, k$ (termwise dominating);

(ii) $A<B$ if $\max (A)<\min (B)$ (global dominating);

(iii) $A \lessdot B$ if $(A-B)<(B-A)$, where $A^{\prime}-B^{\prime}$ stands for the set difference $\left\{i^{\prime}: A^{\prime} \ni i^{\prime} \notin B^{\prime}\right\}$ (global dominating after cancelations);

(iv) $A \triangleright B$ if $A-B \neq \emptyset$, and $B-A$ can be expressed as a disjoint union of nonempty subsets $B^{\prime}, B^{\prime \prime}$ so that $B^{\prime}<(A-B)<B^{\prime \prime}$ (splitting).

Relations (iii) and (iv) give rise to two important notions introduced by Leclerc and Zelevinsky in [3] (where these notions appear in characterizations of quasi-commuting flag minors of a generic $q$-matrix).

Definition. Sets $A, B \subseteq[n]$ are called strongly separated (from each other) if $A \lessdot B$ or $B \lessdot A$ or $A=B$. Sets $A, B \subseteq[n]$ are called weakly separated if either they are strongly separated, or $A \triangleright B$ and $|A| \geq|B|$, or $B \triangleright A$ and $|B| \geq|A|$. Accordingly, a collection $\mathcal{F} \subseteq 2^{[n]}$ is called 
strongly (resp., weakly) separated if any two of its members are such. For brevity, we refer to strongly and weakly separated collections as $s$-collections and $w$-collections, respectively.

Consider a set-system $\mathcal{D} \subseteq 2^{[n]}$, referring to it as a ground collection, or a domain. Our interest is focused on the situation when $\mathcal{D}$ possesses the property of purity with respect to (strongly or weakly) separated collections, which means the following.

Definition. We say that $\mathcal{D}$ is s-pure if all (inclusion-wise) maximal s-collections in $\mathcal{D}$ have the same cardinality, which in this case is called the s-rank of $\mathcal{D}$ and denoted by $r^{s}(\mathcal{D})$. Similarly, we say that $\mathcal{D}$ is $w$-pure if all maximal w-collections in $\mathcal{D}$ have the same cardinality, called the $w$-rank of $\mathcal{D}$ and denoted by $r^{w}(\mathcal{D})$.

(The term "purity" is often used for complexes in which all maximal cells have the same dimension. In our case, we can interpret each s-collection (resp., w-collection) as a cell, forming an abstract simplicial complex with $\mathcal{D}$ regarded as the set of 0 -dimensional cells. This justifies the names "s-pure" and "w-pure".)

Leclerc and Zelevinsky [3] proved that the full domain (Boolean cube) $\mathcal{D}=2^{[n]}$ is s-pure and conjectured that $2^{[n]}$ is w-pure as well (in which case there would be $\left.r^{w}\left(2^{[n]}\right)=r^{s}\left(2^{[n]}\right)=\frac{n(n+1)}{2}+1\right)$. A sharper version of this conjecture deals with $\omega$-chamber sets $X \subseteq[n]$ for a permutation $\omega$ on $[n]$, where $X$ obeys the condition:

$$
\text { if } i<j, \omega(i)<\omega(j) \text {, and } j \in X \text {, then } i \in X \text {. }
$$

They conjectured that the domain $\mathcal{D}(\omega)$ formed by the $\omega$-chamber sets is w-pure (in our terms), with the w-rank equal to $|\operatorname{Inv}(\omega)|+n+1$. Here $\operatorname{Inv}(\omega)$ denotes the set of inversions of $\omega$ (the pairs $(i, j)$ in $[n]$ such that $i<j$ and $\omega(i)>\omega(j))$, and the number $|\operatorname{Inv}(\omega)|$ is called the length of $\omega$. For the longest permutation $\omega_{0}$ (where $\omega_{0}(i)=n-i+1$ ), we have $\mathcal{D}\left(\omega_{0}\right)=2^{[n]}$. 
The above conjecture was proved affirmatively in [2]. The key part consisted in proving the w-purity of $2^{[n]}$; based on this, the result was then shown for an arbitrary permutation $\omega$, and more.

Theorem 1.1 ([2]). The full domain $2^{[n]}$ is w-pure. As a consequence, the following domains $\mathcal{D}$ are $w$-pure as well:

(i) $\mathcal{D}=\mathcal{D}(\omega)$ for any permutation $\omega$ on $[n]$;

(ii) $\mathcal{D}=\mathcal{D}\left(\omega^{\prime}, \omega\right)$, where $\omega^{\prime}, \omega$ are two permutations on [n] with $\operatorname{Inv}\left(\omega^{\prime}\right) \subset \operatorname{Inv}(\omega)$, and $\mathcal{D}\left(\omega^{\prime}, \omega\right)$ is formed by the $\omega$-chamber sets $X \subseteq[n]$ satisfying the additional condition: if $i<j, \omega^{\prime}(i)>\omega^{\prime}(j)$, and $i \in X$, then $j \in X ;$ furthermore, $r^{w}\left(\mathcal{D}\left(\omega, \omega^{\prime}\right)\right)=|\operatorname{Inv}(\omega)|-\left|\operatorname{Inv}\left(\omega^{\prime}\right)\right|+n+1 ;$

(iii) $\mathcal{D}=\Delta_{n}^{m^{\prime}, m}:=\left\{X \subseteq[n] ; m^{\prime} \leq|X| \leq m\right\}$ for any $m^{\prime} \leq m$; furthermore, $r^{\omega}\left(\Delta_{n}^{m^{\prime}, m}\right)=\left(\begin{array}{c}n+1 \\ 2\end{array}\right)-\left(\begin{array}{c}n-m+1 \\ 2\end{array}\right)-\left(\begin{array}{c}m^{\prime}+1 \\ 2\end{array}\right)+1$ (which turns into $m(n-m)+1$ when $m^{\prime}=m$ ).

Note that (ii) generalizes (i) since $\mathcal{D}(\omega)=\mathcal{D}(\mathrm{id}, \omega)$, where id is the identical permutation $(\operatorname{id}(i)=i)$. The domain $\Delta_{n}^{m^{\prime}, m}$ in (iii), generalizes the Boolean simplex (or discrete Grassmannian) $\Delta_{n}^{m}:=\Delta_{n}^{m^{\prime}, m}$. The domains in cases (i), (ii) are s-pure as well, and the w- and s-ranks are equal; see [2]. (Note that in general a domain $\mathcal{D}$ may be w-pure but not s-pure (e.g., for $\mathcal{D}=\Delta_{5}^{2}$ ), and vice versa; also when both w-and s-ranks exist, they may differ.) Using simple observations from [3], one can reduce case (iii) to $2^{[n]}$ as well. In its turn, the proof of w-purity for $2^{[n]}$ given in [2] is direct and essentially relies on a mini-theory of generalized tilings developed in [1].

Another proof for cases (i), (iii) in Theorem 1.1 was given by Oh et al. [4], using a machinery of plabic graphs and alternating strand diagrams elaborated in [5]. 
The purpose of this note is to show that the w-purity for $2^{[n]}$ implies the w-purity for a wider class of domains. They are described in terms of lattice paths in the so-called steep ladder diagrams in the plane (defined in Section 3). Our method of proof for "ladder domains" $\mathcal{D}$ borrows an idea used in [2] for domains $\mathcal{D}(\omega)$ as in Theorem 1.1(i). More precisely, we will consider some w-pure domain $\mathcal{L}$ that includes $\mathcal{D}$ and construct a certain w-collection $\mathcal{C} \subset \mathcal{L}$. This $\mathcal{C}$, called a checker for $\mathcal{D}$, has the property that for any $X \in \mathcal{L}-\mathcal{C}$, the following are equivalent: (a) $X$ belongs to $\mathcal{D}$, and (b) $X$ is weakly separated from $\mathcal{C}$. Then for any maximal w-collection $\mathcal{F}$ in $\mathcal{D}, \mathcal{F} \cup \mathcal{C}$ is a maximal w-collection in $\mathcal{L}$, and now the w-purity of $\mathcal{D}$ follows from that of $\mathcal{L}$.

Section 2 describes properties of checkers needed to us. Section 3 introduces domains generated by steep ladder diagrams, gives illustrations and proves the w-purity for these domains.

Additional terminology. An interval in $[n]$ is a set of the form $\{p, p+1, \ldots, q\}$, and a co-interval is the complement of an interval to $[n]$. For $p \leq q$, we denote by $[p \ldots q]$ the interval $\{p, p+1, \ldots, q\}$.

By a path in a directed graph, we mean a sequence $P=\left(v_{0}, e_{1}, v_{1}\right.$, $\left.\ldots, e_{k}, v_{k}\right)$, where each $e_{i}$ is an edge connecting vertices $v_{i-1}$ and $v_{i}$. An edge $e_{i}$ is called forward (backward) if it goes from $v_{i-1}$ to $v_{i}$ (resp., from $v_{i}$ to $\left.v_{i-1}\right)$, and we write $e_{i}=\left(v_{i-1}, v_{i}\right)$ (resp., $\left.e_{i}=\left(v_{i}, v_{i-1}\right)\right)$. The path is called directed if all its edges are forward. When it is not confusing, we may use notation for $P$ via vertices, writing $P=v_{0} v_{1} \ldots v_{k}$.

\section{Checkers}

In fact, the concept of "checkers" mentioned in the Introduction is applicable wider, due to the following procedure of constructing pure domains. It is convenient to be described in graph theoretic terms, as follows. Let us associate to a domain $\mathcal{D} \subseteq 2^{n}$ the undirected graph $G_{\mathcal{D}}=(V, E)$ whose vertices are the elements of $\mathcal{D}$ and whose edges are 
the weakly (resp., strongly) separated pairs $A, B \in \mathcal{D}, A \neq B$. Then each w-collection (resp., s-collection) in $\mathcal{D}$ corresponds to a clique of $G_{\mathcal{D}}$, a subset of vertices such that any two of them are adjacent (connected by edge) in $G_{\mathcal{D}}$. Accordingly, the w-purity (resp., s-purity) means that all maximal cliques in $G_{\mathcal{D}}$ have the same size.

In general, we can consider an arbitrary undirected graph $G=(V, E)$ and a vertex subset $D \subseteq V$ (playing the role of "domain"). We write $G[D]$ for the subgraph of $G$ induced by $D$ (i.e., the subgraph ( $\left.D, E^{\prime}\right)$ with $E^{\prime}$ maximal). We say that $D$ is pure (w.r.t. cliques) if all maximal cliques in $G[D]$ have the same size.

Lemma 2.1. Let $C$ be a clique in a graph $G=(V, E)$ and let $C^{\prime} \subseteq C$. Define $D$ to be the set of vertices $v$ of $G$ such that $v \notin C-C^{\prime}$ and $\{v\} \cup C$ is a clique. Suppose that the whole set Vis pure. Then D is pure as well.

Proof. Consider a maximal clique $X$ in $G[D]$. Then $X \cap C=C^{\prime}$, and $Y:=X \cup C$ is a clique of $G$. Moreover, $Y$ is a maximal clique of $G$. Indeed, suppose that there is a vertex $v \notin Y$ such that $Y \cup\{v\}$ is a clique. Then $v \notin C$ and $\{v\} \cup C$ is a clique. Hence $v$ belongs to $D$. Moreover, we have $v \in X$, contradicting the maximality of $X$. Now the purity of $D$ follows from that of $V$ and the equality $|Y|=|X|+\left|C-C^{\prime}\right|$.

Corollary 2.2. Let $\mathcal{L} \subseteq 2^{[n]}$ be a w-pure domain. Let $\mathcal{C} \subseteq \mathcal{L}$ be a weakly separated collection and let $\mathcal{C}^{\prime} \subseteq \mathcal{C}$. Define $\mathcal{D}_{\mathcal{C}, \mathcal{C}^{\prime}}^{\mathcal{L}}$ to be the set of $X \in \mathcal{L}$ such that $X \notin \mathcal{C}-\mathcal{C}^{\prime}$ and $X$ is weakly separated from $\mathcal{C}$. Then the domain $\mathcal{D}_{\mathcal{C}, \mathcal{C}^{\prime}}^{\mathcal{L}}$ is w-pure. A similar assertion is valid for the strong separation. 
When a domain $\mathcal{D} \subseteq \mathcal{L}$ is representable in the form $\mathcal{D}_{\mathcal{C}, \mathcal{C}^{\prime}}^{\mathcal{L}}$, where $\mathcal{L}$ and $\mathcal{C}$ are as in the corollary, and $\mathcal{C}^{\prime}=\mathcal{D} \cap \mathcal{C}$, we say that $\mathcal{C}$ is a checker for $\mathcal{D}$ within $\mathcal{L}$ (regarding either w- or s-purity). If we take as $\mathcal{L}$ the entire set $2^{n}$, we abbreviate $\mathcal{D}_{\mathcal{C}, \mathcal{C}^{\prime}}^{\mathcal{L}}$ to $\mathcal{D}_{\mathcal{C}, \mathcal{C}^{\prime}}$.

It should be noted that all particular domains whose purity has been known to us so far are just checker-possessing ones within $2^{n}$. In light of this, one may ask: whether every w-pure domain $\mathcal{D} \subset 2^{[n]}$ has a checker within $2^{n}$ ? However, this is not so, as can be shown by a rather simple counterexample.

For completeness of our description, we now outline explicit constructions of checkers for the domains exposed in Theorem 1.1.

Example 1. Consider the domain $\Delta_{n}^{m^{\prime}, m}$ as in case (iii) of this theorem, where $m^{\prime} \leq m$. Let $\mathcal{C}$ consist of all intervals of size $\geq m$ and all co-intervals of size $\leq m^{\prime}$. One can check that $\mathcal{C}$ is a w-collection. Also one can check that $\mathcal{C}$ is weakly separated from each member of $\Delta_{n}^{m^{\prime}, m}$, and that $X \in 2^{[n]}-\Delta_{n}^{m^{\prime}, m}$ together with the weak separation of $X$ from $\mathcal{C}$ is possible only if $X \in \mathcal{C}$ (cf. [3], Lemma 3.8). Therefore, $\mathcal{C}$ is a checker for $\Delta_{n}^{m^{\prime}, m}$, and we have $\Delta_{n}^{m^{\prime}, m}=\mathcal{D}_{\mathcal{C}, \mathcal{C}^{\prime}}$, where $C^{\prime}$ is formed by the intervals of size $m$ and the co-intervals of size $m^{\prime}$.

Example 2. For a permutation $\omega$ on $[n]$, consider the domain $\mathcal{D}(\omega)$ consisting of the $\omega$-chamber sets (defined by (1.2)). As is shown in ([2], Theorem 2.1), $\mathcal{D}(\omega)$ has as a checker the following set-system:

$$
\mathcal{C}(\omega):=\left\{\omega^{-1}[k] \cap[j \ldots n]: k, j \in[n]\right\} \bigcup\{\emptyset\}
$$

(where possible repeated sets are ignored and where $\omega^{-1}[k]$ stands for $\{i: \omega(i) \in[k]\})$. This implies that $\mathcal{D}(\omega)=\mathcal{D}_{\mathcal{C}(\omega), \mathcal{C}^{\prime}}$ with $\mathcal{C}^{\prime}:=\left\{\omega^{-1}[k]: k \in[n]\right\}$. 
Example 3. Consider the domain $\mathcal{D}\left(\omega^{\prime}, \omega\right)$ defined in case (ii) of Theorem 1.1. It follows from a description in ([2], Section 7) that $\mathcal{D}\left(\omega^{\prime}, \omega\right)$ has as a checker the set-system

$$
\mathcal{C}(\omega) \cup\left\{\left(\omega^{\prime}\right)^{-1}[k] \cap[j]: k, j \in[n]\right\},
$$

where $\mathcal{C}(\omega)$ is defined as in the previous example.

(Note that verifications of the checkers in Examples 2 and 3 are not straightforward; in particular, a proof for $\mathcal{D}\left(\omega^{\prime}, \omega\right)$ in [2] uses a machinery of generalized tilings. It seems that the w-purity of $\mathcal{D}\left(\omega^{\prime}, \omega\right)$ is not implied by results in [4].)

\section{Steep Ladder Diagrams}

In this section, we define ladder diagrams and associate to them lattice paths and domains. Then we present a class of ladder diagrams that generate $\mathrm{w}$-pure domains.

1. By the (full square) grid we mean the directed graph $\Gamma$ whose vertices are the points in $\mathbb{Z}^{2}$ and whose edges are the unit-length segments directed up or to the right. So each vertex (point) $(i, j)$ has one outgoing horizontal edge $((i, j),(i+1, j))$, denoted by hor $_{i, j}$, and one outgoing vertical edge $((i, j),(i, j+1))$, denoted by $\operatorname{vert}_{i, j}$.

Each finite directed path $P$ in $\Gamma$ beginning at the origin $(0,0)$ encodes a finite set $S(P)$ as follows:

(3.1) for $P=v_{0} v_{1} \ldots v_{k}$ with $v_{0}=(0,0), S(P)$ consists of the elements $i \in[k]$ such that the edge $\left(v_{i-1}, v_{i}\right)$ of $P$ is horizontal.

In particular, the set of directed paths $P$ of length $n$ is bijective to $2^{[n]}$, and the set of directed paths $P$ ending at $(m, n-m)$ is bijective to $\Delta_{n}^{m}$ (where $P$ begins at $(0,0)$ ). 
We consider a certain finite part of $\Gamma$. It is determined by a sequence $\lambda=\left(\lambda_{0}, \lambda_{1}, \ldots, \lambda_{k}\right)$ of weakly decreasing nonnegative integers, i.e., $\lambda_{0} \geq \lambda_{1} \geq \ldots \geq \lambda_{k} \geq 0$ (a $(k+1)$-partition $)$. Define $m:=\lambda_{0}$ and $n:=m+k$. The subgraph $\Gamma_{\lambda}=\left(V_{\lambda}, E_{\lambda}\right)$ of $\Gamma$ induced by the set of vertices

$$
V_{\lambda}:=\bigcup_{j=0}^{k}\left\{(i, j): 0 \leq i \leq \lambda_{j}\right\}
$$

is called the ladder determined by $\lambda$. Its north-east boundary $L=L_{\lambda}$ is formed by a (non-directed) path from $(m, 0)$ to $(0, k)$ in which the vertical (horizontal) edges are traversed in the forward (resp., backward) direction. Two examples are drawn in Figure 1 where the paths $L_{\lambda}$ are indicated in bold (omitting directions of edges).

Clearly for each vertex $v$ of $\Gamma_{\lambda}$, any directed path $P$ in $\Gamma$ going from $(0,0)$ to $v$ is entirely contained in $\Gamma_{\lambda}$. Also, the set $S(P)$ does not change under extending $P$ from the end by any number of vertical edges. We define:

(3.2) (i) $T_{\lambda}$ to be the set of vertices $(i, j)$ in $L_{\lambda}$ that are "seen from north and from east", or "forming outer corners", i.e., such that neither hor $_{i, j}$ nor vert $_{i, j}$ belongs to $\Gamma_{\lambda}$;
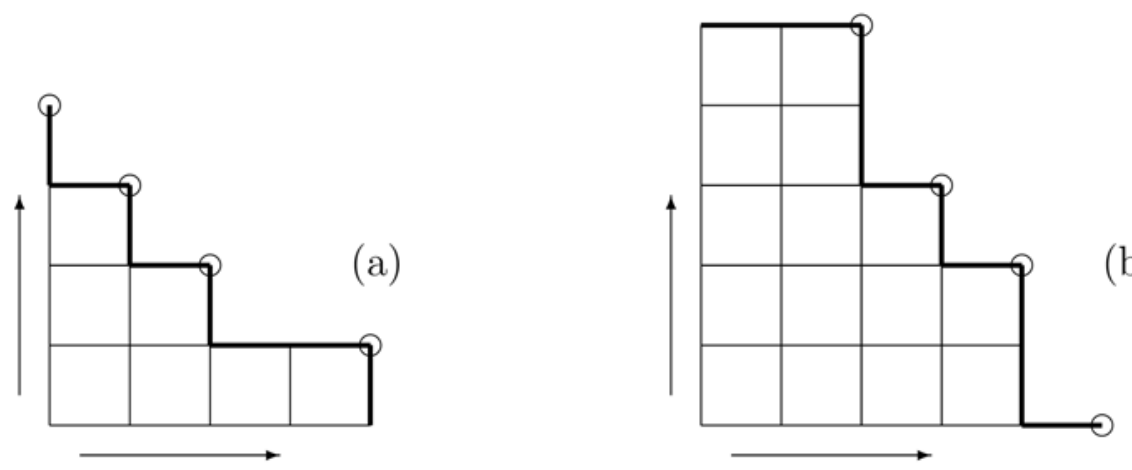

Figure 1. (a) $\Gamma_{4,4,2,1,0}$; (b) $\Gamma_{5,4,4,3,2,2}$. 
(ii) $\mathcal{P}_{\lambda}$ to be the set of directed paths from $(0,0)$ to $T_{\lambda}$;

(iii) $\mathcal{D}_{\lambda}$ to be the collection $\left\{S(P): P \in \mathcal{P}_{\lambda}\right\}$.

(In Figure 1, the set $T_{\lambda}$ is indicated by circles.) The domain $\mathcal{D}_{\lambda}$ is just of interest to us. Note that the map $P \mapsto S(P)$ is injective on $\mathcal{P}_{\lambda}$.

Example 4. When $k=m$ and $\lambda_{i}=k-i$ for $i=0, \ldots, k$ (see Figure 2(a)), we have $\mathcal{D}_{\lambda}=2^{[k]}$.

Example 5. When $\lambda_{0}=\lambda_{1}=\ldots=\lambda_{k}(=m)$ (see Figure 2(b)), $\Gamma_{\lambda}$ spans the rectangle between $(0,0)$ and $(m, k), T_{\lambda}$ consists of the unique vertex $(m, k)$, and $\mathcal{D}_{\lambda}=\Delta_{m+k}^{m}$. This ladder is denoted by $\Gamma^{m, k}$.

Example 6 (Generalizing Examples 4, 5). Let $\lambda_{0}=\lambda_{1}=\ldots=\lambda_{k}(=m)$ and $\lambda_{j}=\lambda_{j-1}-1$ for $j=k^{\prime}+1, \ldots, k$ (see Figure $2(\mathrm{c})$ ). Then $\mathcal{D}_{\lambda}=\Delta_{n}^{m^{\prime}, m}$ for $n:=m+k^{\prime}$ and $m^{\prime}:=m+k^{\prime}-k$.
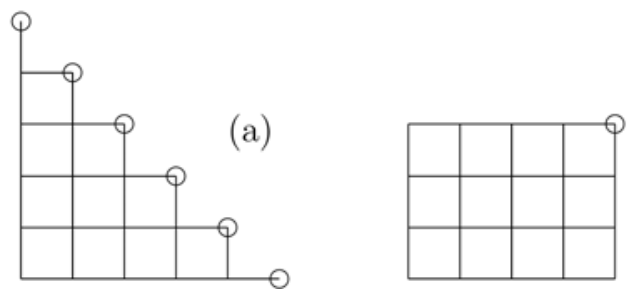

(b)

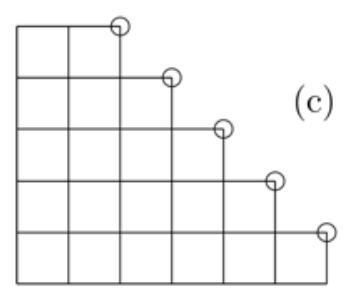

Figure 2. (a) $\lambda=(5,4,3,2,1,0), \mathcal{D}_{\lambda}=2^{[5]}$; (b) $\lambda=(4,4,4,4), \Gamma_{\lambda}=\Gamma^{4,3}$, $\mathcal{D}_{\lambda}=\Delta_{7}^{4}$; (c) $\lambda=(6,6,5,4,3,2), \mathcal{D}_{\lambda}=\Delta_{7}^{2.6}$.

2. Next we specify a class of ladder diagrams and then prove that the domains generated by these ladders are w-pure.

Definition. A ladder $\Gamma_{\lambda}$ is called steep if $\lambda_{i} \geq \lambda_{i-1}-1$ for all $i=1, \ldots, k$. The adjective steep will be applied to the partition $\lambda$ as well. 
In particular, the ladders in Examples 4-6 and in Figure 1(b) are steep, but the one in Figure 1(a) is not. The domains $\mathcal{D}_{\lambda}$ in Examples 4-6 are pure by Theorem 1.1, and relying on the w-purity in case (iii) of that theorem, we show the following:

Theorem 3.1. For a steep ladder $\Gamma_{\lambda=\left(\lambda_{0}, \ldots, \lambda_{k}\right)}$, the domain $\mathcal{D}_{\lambda}$ is w-pure.

Remark 1. $\mathcal{D}_{\lambda}$ need not be w-pure when $\Gamma_{\lambda}$ is not steep. Indeed, let $\lambda$ be as in Figure 1(a). Then $T_{\lambda}=\{(0,4),(1,3),(2,2),(4,1)\}$ and $\mathcal{D}_{\lambda}=\{\emptyset, 1,2,3,4,12,13,14,23,24,34,1234,1235,1245,1345,2345\}$. Since the intervals $\emptyset, 1,12,1234,2345$ are weakly separated from any subset in the interval [5], we can consider the rest in $\mathcal{D}_{\lambda}$. It has maximal w-collections of sizes 4 and 5, e.g., $\{2,4,24,1245\}$ and $\{2,3,4,23,34\}$, whence $\mathcal{D}_{\lambda}$ is not w-pure.

Proof of Theorem 3.1. As before, $m$ stands for $\lambda_{0}$, and we set

$$
m^{\prime}:=\lambda_{k} \quad \text { and } \quad n:=k+m^{\prime}
$$

Define the partition $\mu=\left(\mu_{0}, \ldots, \mu_{k}\right)$ by

$$
\mu_{0}:=n \quad \text { and } \mu_{j}:=\mu_{j-1}-1 \text { for } j=1, \ldots, k .
$$

This $\mu$ is as in Example 6 (with $k^{\prime}=0$ ), and we have $\mathcal{D}_{\mu}=\Delta_{n}^{m^{\prime}, n}$. Also

$$
\mu_{k}=n-k=m^{\prime}=\lambda_{k},
$$

and the steepness of $\Gamma_{\lambda}$ implies $\mu_{j} \geq \lambda_{j}$ for all $j$. Therefore, $\Gamma_{\lambda}$ is entirely contained in $\Gamma_{\mu}$, and $\left(m^{\prime}, k\right)$ is a common vertex of these ladders. This gives $\mathcal{D}_{\lambda} \subseteq \Delta_{n}^{m^{\prime}, n}$. Indeed, for $X \in \mathcal{D}_{\lambda}$, if $P$ is the path in $\mathcal{P}_{\lambda}$ with $S(P)=X$, then adding vertical edges to the end of $P$ (if needed), we obtain a path $P^{\prime}$ in $\mathcal{P}_{\mu}$ such that $S\left(P^{\prime}\right)=S(P)$. We call $P^{\prime}$ the extension of $P$ (within $\Gamma_{\mu}$ ). 
By Theorem 1.1(iii), the domain $\Delta_{n}^{m^{\prime}, n}$ is w-pure. We are going to show the w-purity of $\mathcal{D}_{\lambda}$ by constructing a checker for $\mathcal{D}_{\lambda}$ within $\Delta_{n}^{m^{\prime}, n}$; then the result will follow from Corollary 2.2.

To this aim, we first reformulate the weak separation condition in $\mathcal{D}_{\mu}$ in graphic terms as suggested in [6]. We denote $i$-th edge of a path $P$ by $e_{i}^{P}$.

Definition. Two directed paths $P, Q$ in $\mathcal{P}_{\mu}$ are said to be conflicting if

(3.3) there are $1<a<b \leq n$ such that, up to renaming $P$ and $Q$, the edges $e_{a}^{P}, e_{b}^{Q}$ are horizontal, the edges $e_{b}^{P}, e_{a}^{Q}$ are vertical, $e_{a}^{Q}$ is below $e_{a}^{P}$, and $e_{b}^{Q}$ is below $e_{b}^{P}$.

Here for edges $e, e^{\prime}$ leaving vertices $(i, j)$ and $\left(i^{\prime}, j^{\prime}\right)$, respectively, with $i+j=i^{\prime}+j^{\prime}$, we say that $e$ is (located) below $e^{\prime}$ if either $j<j^{\prime}$, or $j=j^{\prime}$ and $e$ is horizontal, whereas $e^{\prime}$ is vertical. A pair $(a, b)$ as in (3.3) is called critical. See the picture.
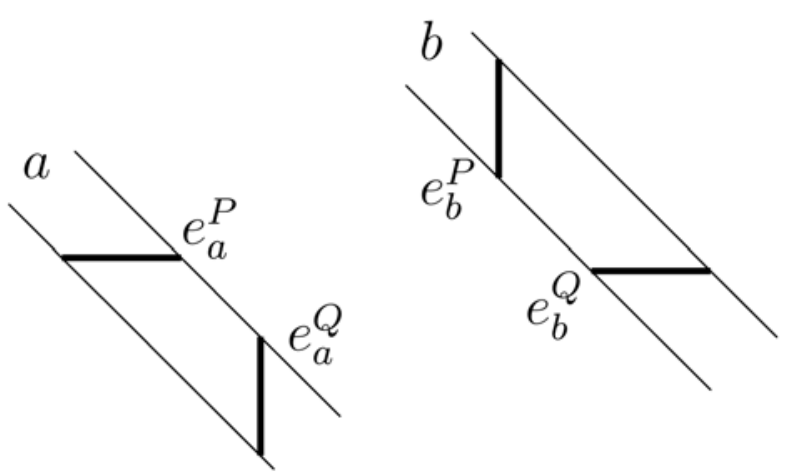

Claim. Let $P, Q \in \mathcal{P}_{\mu}, X=S(P)$, and $Y=S(Q)$. The sets $X, Y$ are weakly separated if and only if $P, Q$ are non-conflicting. 
This fact was established in [6]. To make our description selfcontained (and for the reason that the text of [6] is not accessible at present), we give a proof.

Proof of the Claim. Suppose that $P, Q$ are conflicting, and let $(a, b)$ be a critical pair for them. Then (up to renaming $P, Q), e_{a}^{P}=\operatorname{hor}_{i, j}$, $e_{a}^{Q}=\operatorname{vert}_{i^{\prime}, j^{\prime}}, e_{b}^{P}=\operatorname{vert}_{p, q}, e_{b}^{Q}=\operatorname{hor}_{p^{\prime}, q^{\prime}}$, where $i+j=i^{\prime}+j^{\prime}=a-1$, $i<i^{\prime}, p+q=p^{\prime}+q^{\prime}=b-1, p \leq p^{\prime}$. It follows that $b, c \in Y-X$ and $a \in X-Y$ for some $c<a$ (where $c$ exists because of $|X \cap[a-1]|=$ $\left.i<i^{\prime}=|Y \cap[a-1]|\right)$. Using these relations together with $|X \cap[b]|=$ $|X \cap[b-1]|=p<p^{\prime}+1=|Y \cap[b]|$, it is not difficult to conclude that $X, Y$ cannot be weakly separated.

Conversely, suppose that $X, Y$ are not weakly separated. Let $X-Y$ $=X_{1} \cup X_{2} \cup \ldots \cup X_{\alpha}$ and $Y-X=Y_{1} \cup Y_{2} \cup \ldots \cup Y_{\beta}$, where all $X_{i}, Y_{j}$ are nonempty and, up to renaming $X, Y$, one has $Y_{1}<X_{1}<Y_{2}<X_{2}<\ldots$ (with $<$ defined in (1.1)(ii)). Then one of the following takes place: (i) $\beta \geq 2$ and $\left|X_{1}\right|<\left|Y_{1} \cup Y_{2}\right|$, and (ii) $\alpha, \beta \geq 2$ and $\left|X_{1}\right| \geq\left|Y_{1} \cup Y_{2}\right|$. Define $\quad a:=\min \left(X_{1}\right)$ and $\quad b:=\max \left(Y_{2}\right)$. Then $a<b ; e_{a}^{P}, e_{b}^{Q}$ are horizontal; $e_{a}^{Q}, e_{b}^{P}$ are vertical; and $e_{a}^{Q}$ is below $e_{a}^{P}$ (since $\left.|Y \cap[a-1]|-|X \cap[a-1]|=\left|Y_{1}\right|>0\right)$. Also, in case (i), $e_{b}^{Q}$ is below $e_{b}^{P}$ (since $|Y \cap[b]|-|X \cap[b]|=\left|Y_{1} \cup Y_{2}\right|-\left|X_{1}\right|>0$ ), whence $(a, b)$ is critical and $P, Q$ are conflicting. And in case (ii), for $d:=\min \left(X_{2}\right)$, we have: $b<d ; e_{b}^{Q}, e_{d}^{P}$ are horizontal; $e_{b}^{P}, e_{d}^{Q}$ are vertical; $e_{b}^{P}$ is below $e_{b}^{Q}$ (since $|X \cap[b-1]|>|Y \cap[b-1]|)$; and $e_{d}^{P}$ is below $e_{d}^{Q}$. So $(b, d)$ is critical and $P, Q$ are again conflicting. 
Next we construct the desired checker. Its members are induced by certain paths in $\mathcal{P}_{\mu}$, as follows. For a vertex $(i, j) \in V_{\mu}$, let $H_{i, j}$ be the directed path formed by the vertical path $P_{1}$ from $(0,0)$ to $(0, j)$, followed by the horizontal path $P_{2}$ from $(0, j)$ to $(i, j)$, followed by the vertical path $P_{3}$ from $(i, j)$ to the vertex $\left(i, j^{\prime}\right)$ of $\Gamma_{\mu}$ with $j^{\prime}$ maximum (some of $P_{1}, P_{2}, P_{3}$ may be degenerate). Such an $H_{i, j}$ is called a double hook in $\Gamma_{\mu}$, and we say that it is essential for $\Gamma_{\lambda}$ if the horizontal edge hor $_{i, j}$ does not belong to $\Gamma_{\lambda}$ (note that hor $_{i, j}$ need not belong to $\Gamma_{\mu}$ either). In particular, an essential $H_{i, j}$ ends in $T_{\mu}$ (and therefore belongs to $\left.\mathcal{P}_{\mu}\right)$, and the vertex $(i, j)$ either is not in $\Gamma_{\lambda}$, or belongs to the boundary $L_{\lambda}$ and is "seen from east".

We assert that the collection $\mathcal{C}$ of sets $S(H)$ over all essential double hooks $H$ for $\Gamma_{\lambda}$ is a checker for $\mathcal{D}_{\lambda}$ within $\mathcal{D}_{\mu}$.

Indeed, first of all it is easy to see that any two double hooks are nonconflicting; so $\mathcal{C}$ is a w-collection by the Claim.

Consider an essential double hook $H=H_{i, j}$ and its corresponding concatenation into $P_{1}, P_{2}, P_{3}$ (where $P_{1}, P_{3}$ are vertical and $P_{2}$ is horizontal). Suppose that some path $Q \in \mathcal{P}_{\mu}$ is conflicting to $H$. Then there is a critical pair $(a, b)$ such that (taking into account the construction of $H): e_{a}^{H} \in P_{2}, e_{b}^{H} \in P_{3}, e_{a}^{Q}$ is vertical and lies below $e_{a}^{H}$, and $e_{b}^{Q}$ is horizontal and lies below $e_{b}^{H}$. Let $e_{b}^{H}=\operatorname{vert}_{i^{\prime}, j^{\prime}}$ and $e_{b}^{Q}=\operatorname{hor}_{p, q}$. Since $e_{b}^{H} \in P_{3}$, we have $i^{\prime}=i, j^{\prime} \geq j$, and $i+j \leq i+j^{\prime}=b-1$. At the same time, $p \geq i^{\prime}=i$ ( ince $e_{b}^{Q}$ is below $e_{b}^{H}$ ) and $p+q=b-1$. Now the facts that $\Gamma_{\lambda}$ is steep and that hor $_{i, j}$ is not in $\Gamma_{\lambda}$ imply that the edge $e_{b}^{Q}$ is not in $\Gamma_{\lambda}$ either. This means that $Q$ cannot be the extension of any path in $\mathcal{P}_{\lambda}$, and hence $S(Q)$ is not in $\mathcal{D}_{\lambda}$. 
Conversely, suppose that a path $Q \in \mathcal{P}_{\mu}$ is not the extension of any path in $\mathcal{P}_{\lambda}$. Let $e=\operatorname{hor}_{p, q}$ be the last horizontal edge of $Q$. Then $e$ does not belong to $\Gamma_{\lambda}$. Let $Q^{\prime}$ be the part of $Q$ from $(0,0)$ to $(p+1, q)$. Two cases are possible.

(i) $Q^{\prime}$ is the concatenation of the vertical path from $(0,0)$ to $(0, q)$ and the horizontal path from $(0, q)$ to $(p+1, q)$. Then $Q$ is nothing else than the double hook $H_{p+1, q}$. Moreover, $Q$ is essential since the vertex $(p+1, q)$ (and therefore the edge hor $\left._{p+1, q}\right)$ is not in $\Gamma_{\lambda}$.

(ii) $Q^{\prime}$ is not as in (i). Let $e^{\prime}=\left(i^{\prime}, j^{\prime}\right)$ be the last vertical edge of $Q^{\prime}$. Then $0<i^{\prime} \leq p$ and $j^{\prime}=q-1$. Take the double hook $H=H_{p, q}$. It is essential since hor $_{p, q}$ is not in $\Gamma_{\lambda}$. Also $(p, q)$ cannot be the endvertex of $H$ (since the fact that $(p+1, q)$ is a vertex of $\left.\Gamma_{\mu} \operatorname{implies}(p, q) \notin T_{\mu}\right)$. Therefore, $H$ contains the edge $\operatorname{vert}_{p, q}$. Now define $a:=i^{\prime}+q$ and $b:=p+q+1$. Then $a<b$, the edge $e_{a}^{H}$ is horizontal (namely, $\operatorname{hor}_{i^{\prime}-1, q}$ ) and lies above $e_{a}^{Q}=e^{\prime}=\operatorname{vert}_{i^{\prime}, q-1}$, and the edge $e_{b}^{H}$ is vertical (namely, $\left.\operatorname{vert}_{p, q}\right)$ and lies above $e_{b}^{Q}=\operatorname{hor}_{p, q}$. Hence $Q$ and $H$ are conflicting, implying that the sets $S(Q)$ and $S(H) \in \mathcal{C}$ are not weakly separated.

Thus, $\mathcal{C}$ is indeed a checker for $\mathcal{D}_{\lambda}$ within $\mathcal{D}_{\mu}=\Delta_{n}^{m^{\prime}, n}$ (and $\mathcal{D}_{\lambda}$ is represented as $\mathcal{D}_{C, C^{\prime}}^{\mathcal{L}}$, where $\mathcal{C}^{\prime}$ consists of essential double hooks $H_{i, j}$ with $(i, j)$ contained in the boundary $L_{\lambda}$ and seen from east). Then $\mathcal{D}_{\lambda}$ is w-pure by Corollary 2.2, yielding the theorem.

Remark 2. For a steep partition $\lambda=\left(\lambda_{0}, \ldots, \lambda_{k}\right)$, the w-rank $r^{w}\left(\mathcal{D}_{\lambda}\right)$ is computed as $1+\lambda_{0}+\cdots+\lambda_{k-1}=: \eta_{\lambda}$, which is equal to the number of little squares (inner faces) in $\Gamma_{\lambda}$ plus the number $\left|T_{\lambda}\right|$ of outer corners (cf. (3.2) (i)). To see this, take a vertex $(i, j) \in V_{\lambda}$ that either 
belongs to $T_{\lambda}$ or is the south-east vertex of a little square in $\Gamma_{\lambda}$. We associate to $(i, j)$ the directed path $R_{i, j}$ in $\Gamma_{\lambda}$ that is the concatenation of three paths going, respectively, from $(0,0)$ to $(0, j)$, from $(0, j)$ to $(i, j)$, from $(i, j)$ to $\left(i, j^{\prime}\right)$ with $j^{\prime}$ maximum, and in case $\left(i, j^{\prime}\right) \notin T_{\lambda}$ (i.e., when $i<\lambda_{k}$ and $\left.j^{\prime}=k\right), R_{i, j}$ is extended by the path from $(i, k)$ to $\left(\lambda_{k}, k\right)$. Let $\mathcal{R}$ be the set of such paths; then $|\mathcal{R}|=\eta_{\lambda}$. A routine verification shows that the paths in $\mathcal{R}$ (more precisely, their extensions in $\Gamma_{\mu}$ ) are not conflicting. Also any other path $Q \in \mathcal{P}_{\lambda}$ is conflicting to some path in $\mathcal{R}$. (Such a $Q$ must have consecutive edges vert ve $_{p-1}$ and hor $_{p, q}$ with $p>0$ and $q<k$; therefore, $Q$ is conflicting to $R_{p, q}$.) So $\{S(R): R \in \mathcal{R}\}$ is a maximal w-collection in $\mathcal{D}_{\lambda}$.

In conclusion, it is reasonable to ask: what is the behaviour of ladder diagrams with respect to the strong separation relation? We know (due to [3], Section 4) that the diagrams generating Boolean cubes (illustrated in Example 4) are such. On the other hand, discrete Grassmannians (generated by diagrams as in Example 5) are not s-pure in general. For example, $\Delta_{5}^{2}$ (mentioned in the Introduction) contains maximal s-collections of different sizes, e.g., $\{12,13,15,35,45\}$ and $\{12,13,23,34$, $35,45\}$. One more bad example is generated by the steep partition $\lambda=(2,2,2,1,1,0)$; here $\mathcal{D}_{\lambda}$ has maximal s-collections with different sizes $\{1,12,13,14,34,4,5\}$ and $\{1,2,12,23,24,34,4,5\}$.

However, in spite of seemingly poor behaviour of ladder diagrams with respect to the s-purity, the strong separation relation for the Boolean cube can be reformulated in terms of "conflicting paths" in the corresponding ladder diagram, in spirit of what was done in the weakly separation case. Now, the definition of conflicting paths is modified as follows (cf. the definition in the proof of Theorem 3.1). Let $\mu$ be the partition with $\mathcal{D}_{\mu}=2^{[n]}$. 
Definition. We say that directed paths $P, Q$ in $\mathcal{P}_{\mu}$ are $s$-conflicting if (3.4) there are $1<a<b \leq n$ such that, up to renaming $P$ and $Q$, the edges $e_{a}^{P}, e_{b}^{Q}$ are horizontal, the edges $e_{b}^{P}, e_{a}^{Q}$ are vertical, and $e_{a}^{Q}$ is located below $e_{a}^{P}$.

(Now the conditions on $e_{b}^{Q}$ and $e_{b}^{P}$ are weakened by admitting both cases: $e_{b}^{Q}$ below $e_{b}^{P}$, or $e_{b}^{Q}$ above $e_{b}^{P}$, as illustrated in the picture below.) We call $(a, b)$ an s-critical pair for $P, Q$.

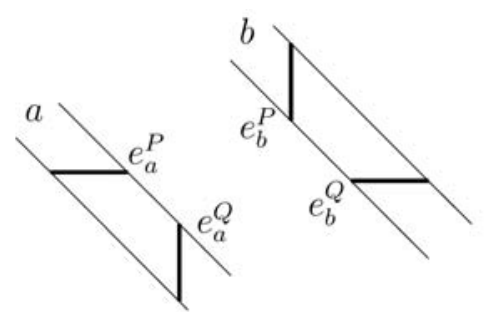

OR

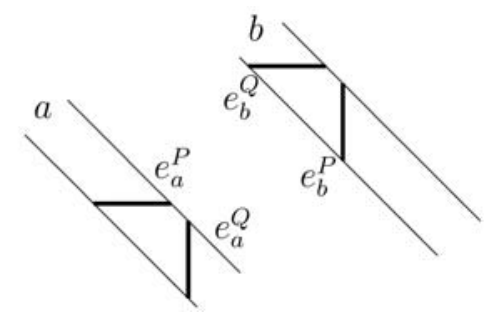

Lemma 3.2. Paths $P, Q \in \mathcal{P}_{\mu}$ are s-conflicting if and only if the sets $X:=S(P)$ and $Y:=S(Q)$ are not strongly separated.

Proof. Suppose that $P, Q$ are conflicting, and let $a, b$ be as in (3.4). The fact that $e_{a}^{Q}$ is below $e_{a}^{P}$ implies the existence of $c \in Y-X$ with $c<a$. We have $c<a<b, c, b \in Y-X$, and $a \in X-Y$, whence $X, Y$ are not strongly separated.

Conversely, suppose that $X, Y$ are not strongly separated. Let $c:=\min (Y-X)$ and $a:=\min (X-Y) ;$ one may assume that $c<a$. Since $X, Y$ are not strongly separated, there exists $b \in Y-X$ such that $b>a$. One can see that the pair $(a, b)$ is s-critical for $P, Q$, as required. 


\section{References}

[1] V. I. Danilov, A. V. Karzanov and G. A. Koshevoy, Plücker environments, wiring and tiling diagrams, and weakly separated set-systems, Adv. Math. 224 (2010), 1-44.

[2] V. I. Danilov, A. V. Karzanov and G. A. Koshevoy, On maximal weakly separated setsystems, J. of Algebraic Combinatorics 32 (2010), 497-531. (Posted ArXiv:0909.1423[math.CO], 2009.)

[3] B. Leclerc and A. Zelevinsky, Quasicommuting families of quantum Plücker coordinates, Amer. Math. Soc. Trans., Ser. 2181 (1998), 85-108.

[4] S. Oh, A. Postnikov and D. E. Speyer, Weak separation and plabic graphs, ArXiv:1109. 4434 [math.CO], 2011.

[5] A. Postnikov, Total positivity, Grassmannians, and networks, ArXiv:math.CO/0609764, 2006.

[6] H. Yoo, On the purity of maximal weakly separated set families, ArXiv:1111.6404[math.CO], 2011. 